\title{
Functions and mechanisms of circular RNAs in cancer radiotherapy and chemotherapy resistance
}

\author{
Chaochu Cui ${ }^{1 \dagger}$, Jianbo Yang ${ }^{2 \dagger}$, Xiao $\mathrm{Li}^{1}$, Dongling Liu', Liwu Fu ${ }^{3 *}$ and Xianwei Wang ${ }^{{ }^{*}}$
}

\begin{abstract}
Circular RNAs (circRNAs), one type of non-coding RNA, were initially misinterpreted as nonfunctional products of pre-mRNA mis-splicing. Currently, circRNAs have been proven to manipulate the functions of diverse molecules, including non-coding RNAs, mRNAs, DNAs and proteins, to regulate cell activities in physiology and pathology. Accumulating evidence indicates that circRNAs play critical roles in tumor genesis, development, and sensitivity to radiation and chemotherapy. Radiotherapy and chemotherapy are two primary types of intervention for most cancers, but their therapeutic efficacies are usually retarded by intrinsic and acquired resistance. Thus, it is urgent to develop new strategies to improve therapeutic responses. To achieve this, clarification of the underlying mechanisms affecting therapeutic responses in cancer is needed. This review summarizes recent progress and mechanisms of circRNAs in cancer resistance to radiation and chemotherapy, and it discusses the limitations of available knowledge and potential future directions.
\end{abstract}

Keywords: circRNAs, Cancer therapy, Sensitivity, Radioresistance, Chemoresistance, Multidrug resistance

\section{Introduction}

Circular RNAs (circRNAs), a type of non-coding RNA (ncRNA), were first reported nearly 4 decades ago. They were initially disregarded as functionless products of pre-mRNA splicing errors $[1,2]$. Since circRNAs have been identified to have a comparable level to their canonical linear counterparts, they have been suggested as pervasive regulatory molecules $[3,4]$.

To date, many circRNAs have been identified and proven to be vital in many diseases, such as cancer [5]. CircRNAs regulate tumor genesis, development, proliferation, migration, invasion and sensitivity to therapy [6].

\footnotetext{
* Correspondence: Fulw@mail.sysu.edu.cn; Wangxianwei1116@126.com ${ }^{\dagger}$ Chaochu Cui and Jianbo Yang contributed equally to this work.

${ }^{3}$ State Key Laboratory of Oncology in South China; Collaborative Innovation Center for Cancer Medicine, Sun Yat-sen University Cancer Center, Guangzhou, China

${ }^{1}$ Henan Key Laboratory of Medical Tissue Regeneration, College of Basic Medical Sciences, Xinxiang Medical University, Xinxiang, Henan, China Full list of author information is available at the end of the article
}

The investigations of long ncRNAs (lncRNAs) and microRNAs (miRNAs), another two types of ncRNAs, have been extensively reported. However, studies of circRNAs in cancer, especially in cancer resistance to radiation and chemotherapy, are still at the nascent stage [7-10].

Inducing tumor cell death is one of the primary effects of radiotherapy and chemotherapy. However, escaping from death, one of the typical characteristics of cancer cells, leads to resistance to therapy, recurrence and poor prognosis of cancers [11]. The mechanisms underlying escape from death include elevated functions of drug efflux pumps, cell stemness, enhanced phagocytosis, and so on [12-16]. It has been increasingly demonstrated that circRNAs manipulate the abovementioned events. This review summarizes the recent progress in the understanding of the mechanisms of cancer resistance potentially related to circRNAs and the functions of circRNAs in radiotherapy and chemotherapy resistance. 
It also discussed the limitations of available knowledge and future potential directions.

\section{Biogenesis and general functions of circRNAs}

The length of conserved circRNAs ranges from hundreds to thousands of nucleotides. They are usually generated from nonsequential back-splicing of pre-mRNA transcripts or back fusion of linear RNAs. CircRNAs with 3' and 5' ends covalently joined to form circular loop structures without free ends are insusceptible to RNase R and exonucleolytic degradation. This grants them higher stability than linear transcripts $[3,4]$. The intergenic circRNAs can be loosely classified into three types (Fig. 1): exonic circRNAs (ecircRNAs), which only contain exons and represent the majority of circRNAs; intronic circRNAs (icircRNAs), which only contain introns; and exon-intron circRNAs (eicircRNAs), which contain both exons and introns [17].

CircRNAs were discovered serendipitously in the early 1970s and disregarded for a long time [1, 2]. Currently, a growing number of circRNAs have been purposely identified with advances in high-throughput sequencing technologies and investigated by bioinformatics methods [18-20]. The classic and major function of circRNAs is serving as molecular sponges of specific miRNAs to regulate mRNA stability and translation [21, 22]. In addition, circRNAs also act as sponges of proteins, as enhancers or coordinators of proteins, mRNAs and DNAs, and as templates for translation (Fig. 1) [17, 23-27].

\section{Actions of circRNAs in cancer}

CircRNAs are promising cancer biomarkers for clinical diagnosis and prognosis because of their high stability and abundance in body fluids (Fig. 1a). The effective circRNAs can be loosely divided into two groups depending on their functions in cancer: suppressors (inhibitors of resistance) and promoters (enhancers of resistance) [6, 28-30]. For instance, circRHOT1 is upregulated in hepatocellular carcinoma (HCC), while circRNA_101505 is downregulated in cisplatin-resistant HCC tissues, and both are related to the survival of HCC patients [23, 31].

\section{Mechanisms mediating radioresistance and chemoresistance potentially related to circRNAs in cancer}

The efficiency of cancer therapy is usually limited by intrinsic and acquired resistance (Fig. 2). CircRNAs widely

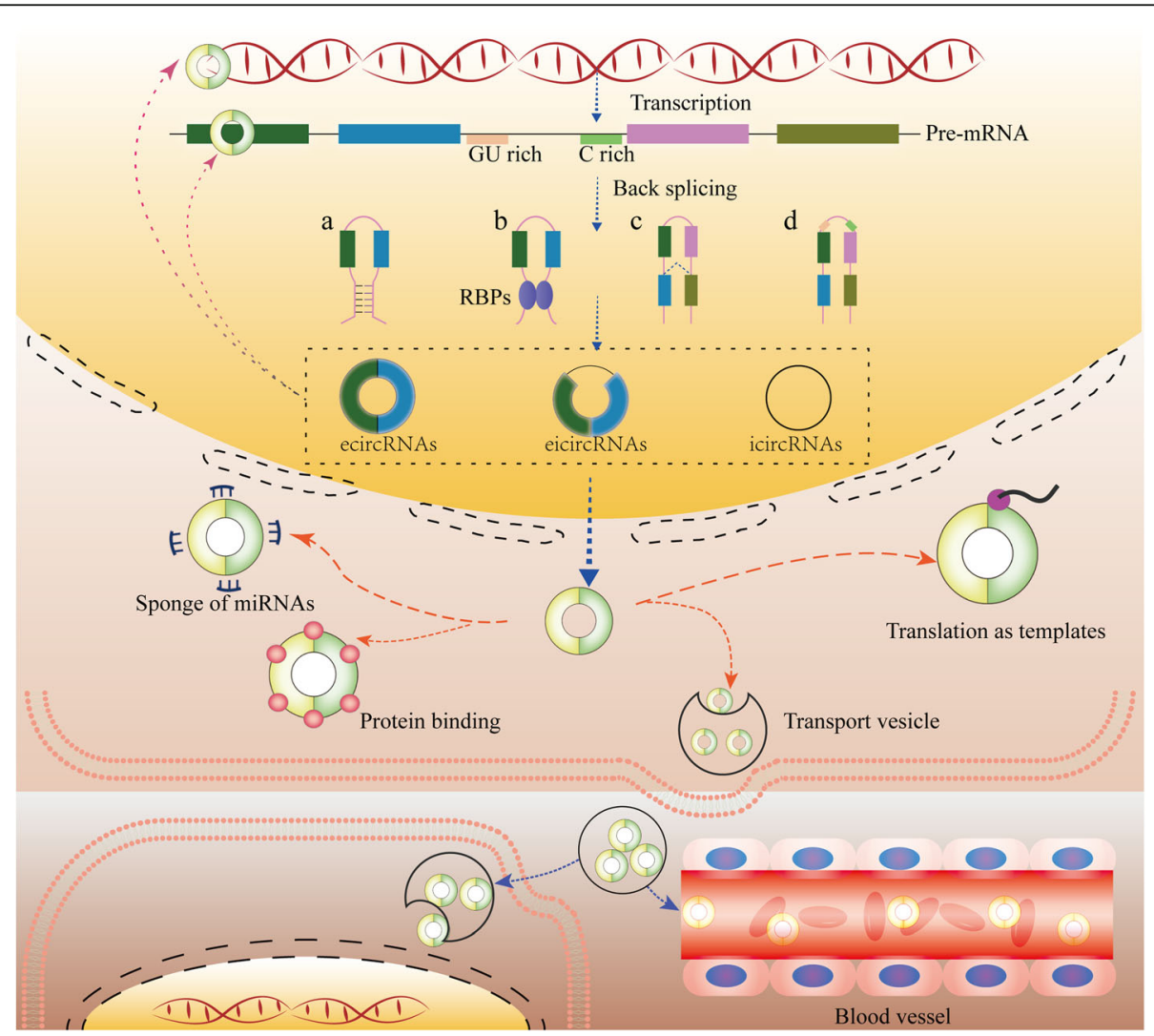

Fig. 1 Biogenesis, distribution and function of circRNAs. CircRNAs are generated from back splicing of pre-mRNAs in different manners (a, b, c and d). CircRNAs can regulate the activities and functions of DNAs, RNAs and proteins in host cells. In addition, they can be secreted into the extracellular space and transported into adjacent cells or body fluids to regulate cell activities. a. Base pairing-dependent circularization; b. RNAbinding protein (RBP)-dependent circularization; c. Lariat-driven circularization; d. GU-rich and C-rich element-dependent circularization 


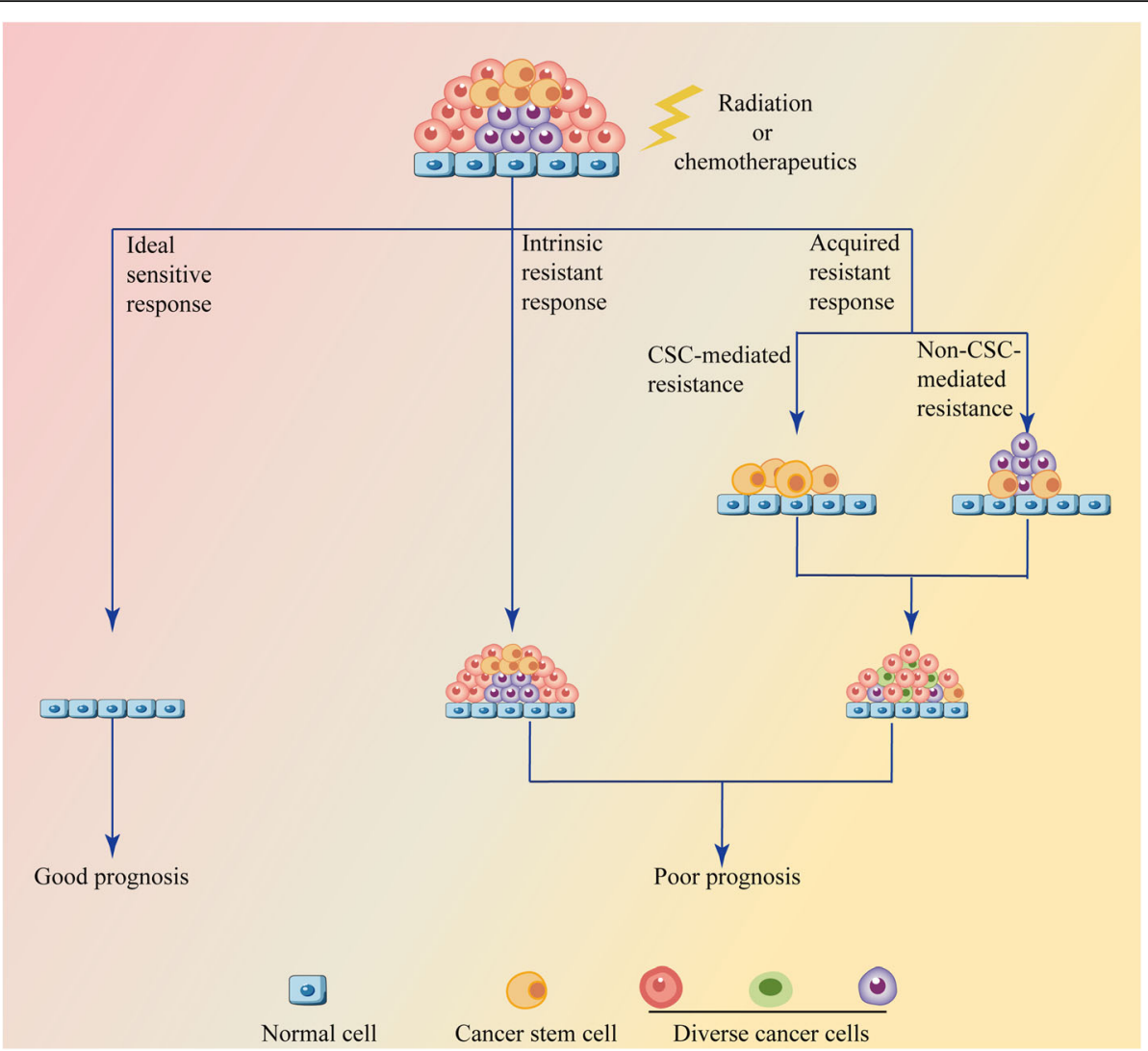

Fig. 2 Responses of cancer cells to radiotherapy and chemotherapy. A completely sensitive response is ideal but rare, while radiotherapy and chemotherapy are usually impeded by acquired or intrinsic resistance. Acquired resistance can be loosely divided into cancer stem cell (CSC)mediated and non-CSC-mediated resistance. CSC-mediated resistance is attributed to the potential ability of CSCs to proliferate and differentiate, which results in regressed tumor recurrence or seeds new tumors in a new place via metastasis. Non-CSC-mediated resistance factors include therapy stress-induced secondary mutations, tumor heterogeneity, altered endoplasmic reticulum (ER) stress, autophagy, drug distribution and metabolism, and so on. The intrinsic resistance mechanisms are similar to those mediating acquired resistance, but the difference is that the characteristics in intrinsic resistance are inherent and not induced by therapy stress

influence cancer characteristics, such as enhanced DNA repair, reduced drug accumulation, target gene amplification and a favorable tumor microenvironment (TME), all of which are important for therapy resistance (Fig. 3).

\section{Increased drug efflux transporters or decreased influx channels}

Reduced drug concentration is a primary cause of chemoresistance. This may result from drug sequestration in intracellular vesicles and compartments, increased drug efflux or decreased drug influx. These changes may be attributed to remodeling of drug channels and transporters, including ATP-binding cassette $(A B C)$ family proteins, solute carriers and volume-regulated anion channels (VRACs) [12, 32-34].

$A B C$ transporters can efficiently transport their corresponding substrates, such as chemotherapeutics, hormones and lipids, to the extracellular compartment, specific organelles and exosomes. Through these processes, the absorption, metabolism and activity of these substrates will be modified. Therefore, ABC transporters are promising targets for reversing multidrug resistance (MDR) [35-37]. Circ-ABCB10 (hsa_circ_ 0008717), derived from a region in the $\mathrm{ABCB} 10$ gene, is highly expressed in breast cancer, epithelial ovarian cancer (OC) and HCC, which correlates with patient survival. However, whether it affects the expression and function of $\mathrm{ABC}$ transporters is worth further investigation $[38,39]$. Silencing of the circRNA PVT1 (circPVT1) decreases the expression of $A B C$ subfamily $B$ member 1 ( $A B C B 1$ or $\mathrm{P}$ gp) and $\mathrm{ABCC} 1[40,41]$. CircSETD3 could bind to miR520 to increase the level of ABCG2, leading to a decreased intracellular accumulation of gefitinib [36].

Platinum-based drugs enter the cell partially through passive diffusion across the cellular membrane and partially through special channels such as VRACs. Leucinerich repeat-containing protein $8 \mathrm{C}$ (LRRC8) heteromers are essential components of VRACs. Chronic myeloid 


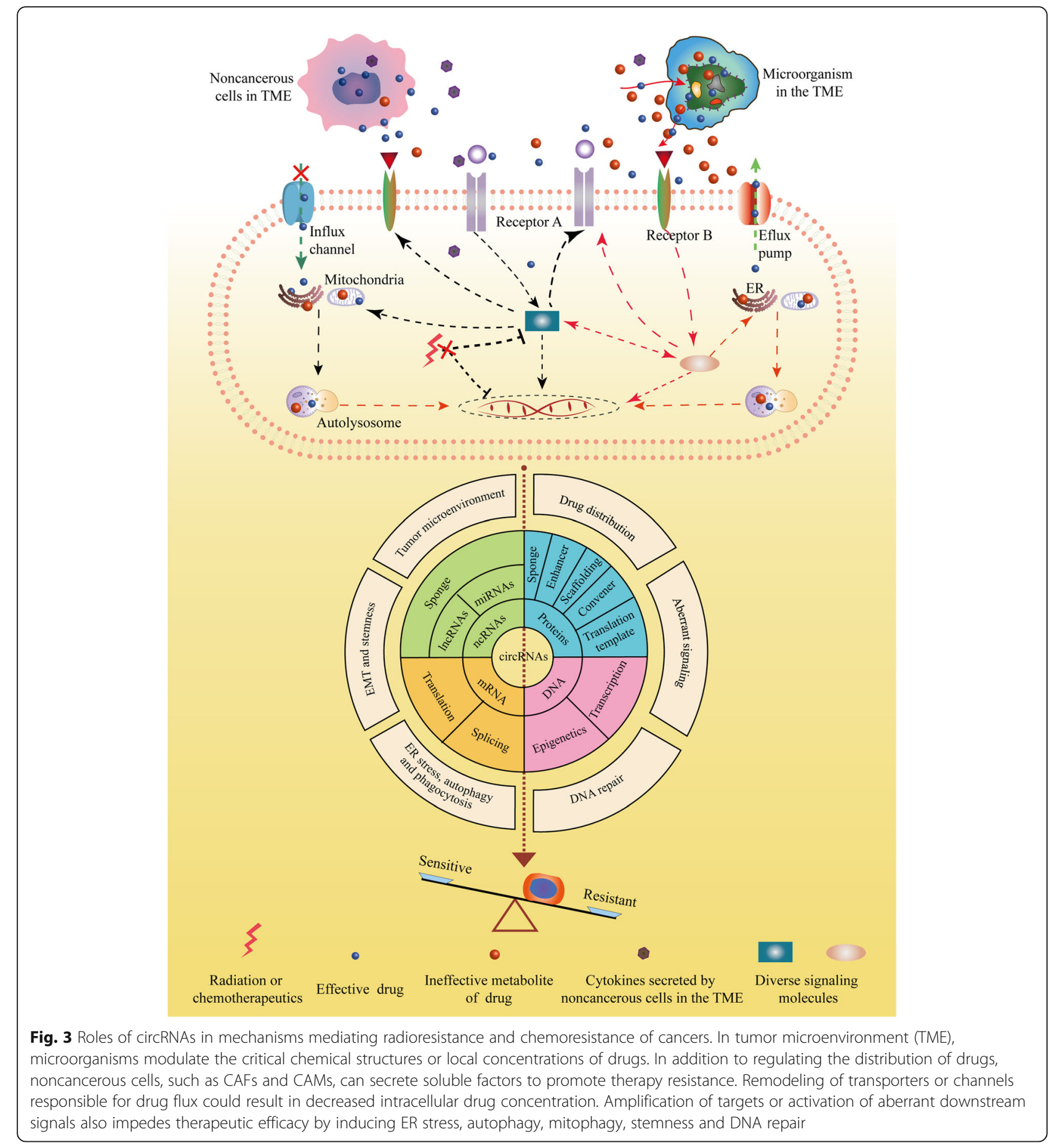

leukemia (CML) cells deficient in LRRC8A or LRRC8D were proved to be more resistant to carboplatin and cisplatin than cells with these proteins $[33,42]$.

\section{Enhanced epithelial-to-mesenchymal transition (EMT) and stemness}

CiRS-7 (CDR1as or CDR1NAT), a cancer suppressor derived from the lncRNA LINC00632 transcripts, is upregulated in osteosarcoma (OS) and esophageal squamous cell carcinoma (ESCC). It is positively associated with cancer progression and promotes EMT by regulating miR-7 and miR-876-5p [43, 44]. EMT is an epigenetic program, through which the cells lose their epithelial phenotypes and gain mesenchymal characteristics. EMT-inducing transcription factors such as twist and snail, contain complementary binding sites to 
promoters of $\mathrm{ABC}$ transporters. They not only enhance EMT but also elevate the expression of $\mathrm{ABC}$ transporters. Silencing of these EMT inducers successfully reverses EMT-related MDR in breast cancer cells [12]. CircSMAD2 is capable of suppressing EMT by reducing miR-629 expression, which is a potential target for enhancing therapy sensitivity [45].

Circ_001680 and circ-NOTCH1 are increased in colorectal cancer $(\mathrm{CRC})$ and gastric cancer $(\mathrm{GC})$, respectively. Both promote the cancer stem cell (CSC) population in cancer $[46,47]$. Failing to eradicate cancer cells that acquire stemness via activation of EMT or senescence is another reason for compromised therapy outcomes in cancer [48-51]. CSCs are capable of being dormant for a very long time to escape from harmful stress, such as radiotherapy and chemotherapy. Once the harmful stress is removed, CSCs may differentiate and proliferate at the primary lesion or metastasize and then invade other organs, leading to regressed tumor recurrence or metastasis $[13,16,52]$.

\section{Copy number alterations of target genes and activation of bypass pathways or downstream signals}

Heterogeneity is another major characteristic of cancer [11]. It promotes cancer resistance to therapy and even retards immunotherapy responses [53]. Heterogeneity indicates that various types of cells are present in an individual tumor, while a specific signal may play a predominant role in different cells and in different stages of cancer [11, 54-58]. Elevation of PD-L1 is a primary obstacle in cancer immunotherapy. CiRS-7 and circRNA002178 were proven to increase the accumulation of PD-L1 at the cell membrane [59-61]. Extrachromosomal DNA (ecDNA) without centromeres will not be averagely transmit to new generations, which is a critical cause for heterogeneity. Amplification of the dihydrofolate reductase gene in ecDNA induces methotrexate resistance [62]. In contrast, the cells will become resistant to EGFR inhibitors if the mutant epidermal growth factor receptor (EGFR) is depleted from ecDNA. This causes the reemergence of tumors following drug withdrawal [63].

PI3K inhibitors are reported to not only induce the activation of the oncogene Myc but also stimulate the amplification of PIK3CA, both of which reduce cancer cell sensitivity [64]. Osimertinib is able to increase the phosphorylation of AXL in EGFR-mutated non-small cell lung cancer (NSCLC) cells, which subsequently activate HER3, MET, EGFR and the corresponding downstream signals. All of these oncological signals reduce the responses of cancers to osimertinib [65]. Similarly, inhibition of PI3K $\alpha$ also increases AXL expression, which induces chemoresistance through transactivation of the EGFR/PKC/mTOR axis independent of PI3K [66].
STAT3 and AKT are strongly associated with carcinogenesis by enhancing the proliferation and reducing the apoptosis of tumor cells [67-69]. The Skp2/AKT axis is capable of inducing the phosphorylation of programmed cell death protein 4 (PDCD4), a protein that promotes the DNA damage response and apoptosis. Blockade of this action favors cancer cells resistant to radiotherapy [15]. Under chemotherapy stress, the feedback activation of STAT3 by the IL-6/JAK1 or FGFR/PI3K pathway prevents cancer cells from undergoing the apoptosis induced by inhibitors of receptor tyrosine kinases (RTKs), such as MEK or KRAS [68]. Hsa_circ_0017250, derived from the AKT gene, encodes a protein that can inactivate AKT and enhance cell sensitivity to radiation [25].

\section{Remodeling of ER stress, autophagy and phagocytosis}

Both activating and inhibiting ER stress can overcome chemoresistance and radioresistance in cancer [70-72]. This indicates that the roles of ER stress in cell fate are multifaceted and context-dependent. ER stress is also a potent inducer of autophagy that manipulates the sensitivity of cancers [73]. For instance, imatinib induces autophagy that facilitates chemoresistance [74]. However, autophagy is also a double-edged sword. Mild autophagy favors cell survival under normal or mild stress conditions, while excessive autophagy leads to cell death. Therapy resistance may be attributed to autophagymediated changes in oxidative stress and autophagy-supplied key metabolites for the maintenance of stemness during dormancy [75-78]. Interestingly, circEIF6 promotes cisplatin-induced autophagy and enhances chemoresistance in GC cells [79].

Following doxorubicin (DOX) treatment, the induced senescent cancer cells can expand, approach and then completely engulf the neighboring cells. The engulfed cells are transported to the lysosomes for degradation, which is similar to autophagy in terms of supplying energy and key metabolites. This reduces chemotherapy responses by mechanisms such as preventing tumor cell death and leads to tumor relapse [14].

\section{Enhanced or weakened DNA repair ability}

Inducing DNA damage is another primary action of radiotherapy and chemotherapy that usually causes cell death. However, DNA repair is able to reduce the sensitivity of cancer cells to therapy. Mutations in the BRCA1 and BRCA2 genes, two critical enzymes for homologous recombination (HR) or mismatch repair (MMR) DNA repair, can promote the progression of breast cancer and OC $[80,81]$. Due to their limited DNA repair ability, cells with certain BRCA1 or BRCA2 mutations are supposed to be sensitive to cell death stimuli such as cisplatin, poly ADP-ribose polymerase (PARP) inhibitors and radiation [82, 83]. However, hsa_circ_0000199 has 
the potential to activate the DNA repair molecule histone family member $\mathrm{X}(\mathrm{H} 2 \mathrm{AX})$, leading to resistance to cisplatin $[81,84]$.

Furthermore, the expression of dynein light chain 1 protein (DYNLL1) is positively correlated with progression-free survival (PFS) in platinum-based chemotherapy in patients with BRCA1-mutated ovarian carcinoma. Once DYNLL1 is inactivated, the nuclease activity of MRE11, a double-strand break repair protein, will be restored to repair errors in DNA, even without BRCA1 coordination. This further confers resistance to platinum drugs and PARP inhibitors in BRCA1-mutant cells [83]. Interestingly, therapeutic stresses such as EGFR/BRAF inhibitors decrease the levels of MMR and HR DNA repair genes, meanwhile increase the errorprone polymerases. These actions prompt adaptive DNA mutability and ultimately restrain chemotherapy efficacy by conferring cancer cell resistance [85].

\section{Favorable tumor microenvironment}

The TME is a complex ecosystem containing the microbiota, an acidic $\mathrm{pH}$, inflammatory factors, matrix metalloproteinases, extracellular matrix (ECM), cancer-associated fibroblasts (CAFs), tumor-associated macrophages (TAMs) and so on. The TME is even different between the primary tumor and the corresponding metastases, which also affects cancer cell sensitivity [11, 86]. Inflammatory factors such as IL-7 can reduce the expression of ABCG2 and resensitize NSCLC cells to cisplatin [87]. The hypoxic TME induces excessive mitochondrial fission in natural killer (NK) cells. This reduces NK cell viability and immunosurveillance [88]. Tumor cell-secreted PRSS (a serine protease) not only promotes angiogenesis and cancer cell invasion but also induces resistance to monoclonal antibodies such as cetuximab and bevacizumab by cleaving them into inactive forms [89]. The microorganisms in the TME decrease the efficacy of chemotherapeutics in various manners. For example, Gammaproteobacteria, a common bacterial species in pancreatic cancer tissues, lead to poor responses by metabolizing gemcitabine into inactive metabolites in cancer cells [90].

Before cancer cells, TAMs will infiltrate into irradiated normal tissues under radiotherapeutic stress. Then, TAMs secrete soluble factors such as inflammatory protein 1 beta to recruit cancer cells, promoting cancer metastasis and recurrence following radiotherapy [91]. Soluble factors from TAMs also have the potential to induce the expression of cytidine deaminase (a metabolism enzyme) in cancer cells, which reduces the efficacy of gemcitabine [90].

Similar to TAMs, CAFs render tumor cells resistant to 5-FU, which is correlated with the poor cumulative survival of GC patients. The responses of lung cancers with podoplanin-positive CAFs to EGFR tyrosine kinase inhibitors (TKIs) are worse than those of lung cancers with podoplanin-negative CAFs [92, 93]. There are many other factors in the TME that can affect drug sensitivity, such as an aberrant vasculature failing to transport drugs. With diverse functions, circRNAs such as ciRS-7 have already been shown to regulate various characteristics of the TME, which has been proven to regulate the sensitivity of several types of cancer to chemotherapy [26, 94-98].

\section{Advances in the understanding of circRNA- mediated radioresistance and chemoresistance in cancer}

Although the underlying mechanisms of therapy resistance have been extensively studied, it is still one of the biggest obstacles in cancer treatment. Recently, circRNAs have attracted intensive interest from oncologists due to their potential functions in tumor biology. There have been dozens of reports on the actions of circRNAs in therapy resistance (Tables 1 and 2), of which only several studies are about the role of circRNAs in radiotherapy responses (Table 1 ).

\section{CircRNAs in cancer radioresistance \\ Glioma}

CircATP8B4 is one of the increased circRNAs (Table 1) in radioresistant glioma, and it sponges miR-766, which is probably the mechanism through which circATP8B4 reduces radiation sensitivity [99]. The blood-brain barrier $(\mathrm{BBB})$ prevents many drugs from entering the brain, leading to a poor response of glioma to chemotherapy. An in vitro study indicated that the circRNA DENND4C (cDENND4C), as an miR-577 sponge, increases mimicked $\mathrm{BBB}$ permeability by reducing the expression of tight junction-related proteins. This allows more DOX across mimicked BBB in vitro, leading to apoptosis of glioma cells [152]. However, whether this phenomenon also exists in vivo has still not been studied. Thus, radiotherapy is still the preferred strategy for brain cancers to date. Three circRNAs generated from the AKT3 gene (hsa_circ_0017250, hsa_circ_0112784 and hsa_circ 0112781) are decreased in glioma tissues. Hsa_circ 0017250, containing a complete ORF, encodes a protein named AKT3-174aa. AKT3-174aa acts as a dominantnegative variant of $\mathrm{AKT}$ and inhibits the phosphorylation of $\mathrm{AKT}^{\mathrm{T} 308}$, thus enhancing the sensitivity of glioma cells to radiation [25].

\section{Nasopharyngeal carcinoma}

In radioresistant nasopharyngeal carcinoma (NPC) cells, 2600 altered circRNAs have been identified, 1042 of which were upregulated and 1558 of which were downregulated. Hsa_circRNA_006660 was further proven to enhance radiosensitivity via miR-1276 [100]. CircRNA_ 
Table 1 Alterations of circRNAs in radiotherapy resistance

\begin{tabular}{|c|c|c|c|c|}
\hline Cancer & Numbers of alterations/name of circRNA & Alteration & Target & References \\
\hline \multirow[t]{3}{*}{ Glioma } & hsa_circ_0017250 & $\downarrow$ & AKT protein & [25] \\
\hline & 48 & $\downarrow$ & - & [99] \\
\hline & 63 & $\uparrow$ & & \\
\hline \multirow[t]{3}{*}{ Nasopharyngeal carcinoma } & 1558 & $\downarrow$ & - & [100] \\
\hline & 1042 & $\uparrow$ & & \\
\hline & circRNA_000543 & $\uparrow$ & miR-9 & [101] \\
\hline Oral squamous cell carcinoma & circATRNL1 & $\downarrow$ & $\operatorname{miR}-23 a-3 p$ & [102] \\
\hline \multirow[t]{4}{*}{ Esophageal cancer } & 17 & $\downarrow$ & Enriched in Wnt signaling pathway & [103] \\
\hline & 57 & $\uparrow$ & & \\
\hline & circVRK1 & $\downarrow$ & miR-624-3p & [104] \\
\hline & circRNA_100367 & $\uparrow$ & miR-217 & [105] \\
\hline Hepatocellular carcinoma & cZNF292 & Increased under hypoxia & SOX9 protein & [106] \\
\hline \multirow[t]{3}{*}{ Colorectal cancer } & 47 & $\downarrow$ & - & [107] \\
\hline & 24 & $\uparrow$ & & \\
\hline & circCCDC66 & $\uparrow$ & miR-338-3p & [108] \\
\hline \multirow[t]{2}{*}{ Cervical cancer } & 76 & $\uparrow$ & - & [109] \\
\hline & 77 & $\downarrow$ & & \\
\hline
\end{tabular}

Notes: $\uparrow$ means increased; $\downarrow$ means decreased; -- means not reported yet

000543 is elevated in NPC tissues and is much higher in radioresistant samples than in radiosensitive samples. Silencing it sensitizes NPC cells to irradiation. It was further demonstrated that circRNA_000543 sponges miR-9 and then increases transcription of platelet-derived growth factor receptor beta (PDGFRB), leading to poorer overall survival of NPC patients [101].

\section{Oral squamous cell carcinoma}

CircATRNL1, derived from the ATRNL1 gene, is one of the dysregulated circRNAs that is reduced in oral squamous cell carcinoma (OSCC) cells, and its level is lower following irradiation. When circATRNL1 is upregulated, the radiosensitivity of OSCC cells is enhanced. This is because it sponges miR-23a-3p to increase the transcription of PTEN and then decreases the phosphorylation of AKT, suggesting that autophagy may be involved [102].

\section{Esophageal cancer}

$\mathrm{Su}$ et al. identified 57 circRNAs that were upregulated and 17 that were downregulated in radioresistant ESCC cells. Wnt signals were revealed to be the most enriched downstream pathways of these altered circRNAs [103, 153]. The level of circRNA_100367 is increased in radioresistant ESCC cells. It binds to miR-217 to promote Wnt3mediated EMT, resulting in reduced sensitivity of cancer cells to radiation [105]. CircVRK1 is decreased in ESCC tissues, and patients with low level of circVRK1 have a poor prognosis. This is probably because circVRK1, as a sponge of miR-624-3p, decreases the PTEN/PI3K- mediated activity of AKT and inhibits EMT, which enhances the sensitivity of ESCC cells to radiotherapy [104].

\section{Hepatocellular carcinoma}

cZNF292 is upregulated in HCC cells under hypoxia independently of HIF-1 $\alpha$. Silencing cZNF292 inhibits radiation-induced $\gamma-\mathrm{H} 2 \mathrm{AX}$ foci formation due to its binding with SOX9 protein to inhibit the activity of the $\beta$-catenin-mediated DNA repair pathway. This ultimately enhances the sensitivity of HCC cells to radiotherapy [106].

\section{Colorectal cancer}

A microarray analysis identified 47 upregulated circRNAs and 24 downregulated circRNAs in 5-FU- and radiation-resistant $\mathrm{CRC}$ cells. Bioinformatics pathway analysis revealed that these circRNAs were related to miRNAs and cancer-associated signals, such as the Wnt pathway [107]. CircCCDC66 is increased in CRC tissues and is much higher in radioresistant tissues than in radiosensitive ones and is associated with decreased levels of miR-338-3p. Since circCCDC66 sponges miR-338-3p, silencing circCCDC66 inhibits cell viability and enhances CRC cell radiosensitization via caspase-3 [108].

\section{Miscellaneous}

It was identified that 61 circRNAs are upregulated and 97 are downregulated in human embryonic kidney (HEK) $293 \mathrm{~T}$ cells following radiation. The circRNAmiRNA-mRNA network analysis revealed that the 
Table 2 Altered circRNAs in chemoresistant cancer cells

\begin{tabular}{|c|c|c|c|c|c|c|}
\hline System & Cancer & $\begin{array}{l}\text { Number of alterations/name } \\
\text { of circRNA }\end{array}$ & Alteration & Target & Related drugs & References \\
\hline \multirow{4}{*}{$\begin{array}{l}\text { Musculoskeletal } \\
\text { system }\end{array}$} & \multirow[t]{4}{*}{ Osteosarcoma } & hsa_circ_0001258 & $\downarrow$ & miR-744-3p & Doxorubicin & {$[110]$} \\
\hline & & Circ-LARP4 & & miR-424 & Cisplatin, doxorubicin & [111] \\
\hline & & has_circ_0004674 & $\uparrow$ & - & Doxorubicin & [112] \\
\hline & & circPVT1 & & & $\begin{array}{l}\text { Methotrexate, doxorubicin, } \\
\text { cisplatin, ifosfamide }\end{array}$ & {$[40]$} \\
\hline \multirow[t]{14}{*}{$\begin{array}{l}\text { Respiratory } \\
\text { System }\end{array}$} & $\begin{array}{l}\text { Metastatic } \\
\text { Nasopharyngeal } \\
\text { carcinoma }\end{array}$ & circCRIM1 & $\uparrow$ & miR-422a & Paclitaxel & [113] \\
\hline & \multirow[t]{13}{*}{ Lung cancer } & 7966 & $\uparrow$ & - & Osimertinib & [114] \\
\hline & & 7538 & $\downarrow$ & & & \\
\hline & & 2909 & $\uparrow$ & - & Paclitaxel & {$[20]$} \\
\hline & & 8372 & $\downarrow$ & & & \\
\hline & & circESRP1 & $\downarrow$ & miR-93-5p & $\begin{array}{l}\text { Doxorubicin, cisplatin, } \\
\text { etoposide }\end{array}$ & {$[115]$} \\
\hline & & circ_0002483 & $\downarrow$ & miR-182-5p & Paclitaxel & [116] \\
\hline & & hsa_circ_0004015 & $\uparrow$ & miR-1183 & Gefitinib & [117] \\
\hline & & circSETD3 & & miR-520 h & & {$[36]$} \\
\hline & & CCDC66 & & - & Cisplatin & [118] \\
\hline & & circ_0076305 & & miR-296-5p & Cisplatin & [119] \\
\hline & & circFGFR1 & & miR-3 81-3p & PD-1 antibody & [120] \\
\hline & & circPVT1 & & miR-145-5p & Cisplatin, pemetrexed & [41] \\
\hline & & ciRS-7 & & - & Pemetrexed, cisplatin & [121] \\
\hline \multirow{5}{*}{$\begin{array}{l}\text { Hematopoietic } \\
\text { system }\end{array}$} & \multirow[t]{5}{*}{ Leukemia } & circ_100053 & $\uparrow$ & - & Imatinib & [122] \\
\hline & & circMYBL2 & & PTBP1 protein & Quizartinib & [24] \\
\hline & & crCPAN3 & & $\begin{array}{l}\text { miR-153-5p and miR- } \\
183-5 p\end{array}$ & Doxorubicin & {$[123,124]$} \\
\hline & & circBA9.3 & & - & $\begin{array}{l}\text { Imatinib, nilotinib, } \\
\text { dasatinib }\end{array}$ & [125] \\
\hline & & circ_0009910 & & $\operatorname{miR}-34 a-5 p$ & Imatinib & {$[126]$} \\
\hline \multirow[t]{12}{*}{$\begin{array}{l}\text { Endocrine } \\
\text { system }\end{array}$} & $\begin{array}{l}\text { Thyroid } \\
\text { carcinoma }\end{array}$ & circEIF6 & $\uparrow$ & miR-144-3p & Cisplatin & [79] \\
\hline & \multirow[t]{3}{*}{$\begin{array}{l}\text { Pancreatic } \\
\text { cancer }\end{array}$} & $\begin{array}{l}\text { chr14: 101402109-101464448+, } \\
\text { chr4: 52729603-52780244+ }\end{array}$ & $\uparrow$ & miR-145 & Gemcitabine & [127] \\
\hline & & 26 & $\uparrow$ & - & & [19] \\
\hline & & 55 & $\downarrow$ & & & \\
\hline & \multirow[t]{2}{*}{ Cervical cancer } & circMTO1 & $\uparrow$ & miR-6893 & Cisplatin & [128] \\
\hline & & hsa_circ_0023404 & & miR-5047 & & [129] \\
\hline & \multirow[t]{6}{*}{ Breast cancer } & circ 0006528 & $\uparrow$ & miR-7 & Doxorubicin & [130] \\
\hline & & ciRS-7 & & miR-7 & Cisplatin, 5-FU, tamoxifen & [131-133] \\
\hline & & circRNA-MTO1 & $\downarrow$ & TRAF4 protein & Monastrol & [134] \\
\hline & & hsa_circ_0025202 & & miR-182-5p & Tamoxifen & [135] \\
\hline & & circBMPR2 & & miR-553 & & [136] \\
\hline & & circKDM4C & & miR-548p & Doxorubicin & [137] \\
\hline \multirow{2}{*}{$\begin{array}{l}\text { Digestive } \\
\text { system }\end{array}$} & Oral carcinoma & hsa_circ_0005379 & $\downarrow$ & - & Cetuximab & [138] \\
\hline & Hepatocellular & circRNA_101505 & $\downarrow$ & miR-103 & Cisplatin & [31] \\
\hline
\end{tabular}


Table 2 Altered circRNAs in chemoresistant cancer cells (Continued)

\begin{tabular}{|c|c|c|c|c|c|c|}
\hline System & Cancer & $\begin{array}{l}\text { Number of alterations/name } \\
\text { of circRNA }\end{array}$ & Alteration & Target & Related drugs & References \\
\hline & Gastric cancer & hsa_circ_0000199 & $\uparrow$ & miR-198 & Cisplatin & [84] \\
\hline & & hsa_circ_0081143 & & miR-646 & & [139] \\
\hline & & circFN1 & & miR-182-5p & & {$[140,141]$} \\
\hline & & circPVT1 & & miR-124-3p & Paclitaxel & [141] \\
\hline & Colorectal & 773 & $\uparrow$ & - & 5-FU and oxaliplatin & {$[142]$} \\
\hline & cancer & 732 & $\downarrow$ & & & \\
\hline & & 34 & $\downarrow$ & & & [29] \\
\hline & & 105 & $\uparrow$ & & & \\
\hline & & circ_001680 & $\uparrow$ & miR-340 & Irinotecan & {$[46]$} \\
\hline & & CiRS-122 & & miR-122 & Oxaliplatin & [143] \\
\hline \multirow[t]{9}{*}{ Urinary system } & Renal cancer & hsa_circ_0035483 & $\uparrow$ & miR-335 & Gemcitabine & [144] \\
\hline & Bladder cancer & hsa_circ_0000285 & $\downarrow$ & - & - & [145] \\
\hline & & ciRS-7 & & miR-1270 & Cisplatin & [95] \\
\hline & & circELP3 & $\uparrow$ & - & - & [96] \\
\hline & Prostate cancer & circRNA17 & $\downarrow$ & miR-181c-5p & Enzalutamide & {$[146]$} \\
\hline & & 588 & $\downarrow$ & - & & [147] \\
\hline & & 278 & $\uparrow$ & & & \\
\hline & & $\begin{array}{l}\text { hsa_circ_0022392 and hsa_ } \\
\text { circ_0000326 }\end{array}$ & $\downarrow$ & - & - & [148] \\
\hline & & $\begin{array}{l}\text { hsa_circ_0001275 and hsa_ } \\
\text { circ_0001721 }\end{array}$ & $\uparrow$ & - & - & \\
\hline \multirow{2}{*}{$\begin{array}{l}\text { Reproductive } \\
\text { system }\end{array}$} & Ovarian cancer & ciRS-7 & $\downarrow$ & miR-1270 & Cisplatin & {$[26]$} \\
\hline & & circCELSR1 & $\uparrow$ & miR-1252 & Paclitaxel & [149] \\
\hline \multirow[t]{2}{*}{ Miscellaneous } & Myeloma & hsa_circ_0007841 & $\uparrow$ & - & - & [150] \\
\hline & Melanoma & ciRS-7 & $\downarrow$ & - & - & [151] \\
\hline
\end{tabular}

Notes: $\uparrow$ means increased; $\downarrow$ means decreased; -- means not reported yet

altered circRNAs were suggested to be involved in EGFR and mTOR signaling, which is worth further studies in cancer cells [154]. Duo et al. identified 76 upregulated and 77 downregulated circRNAs in irradiated HeLa cells. However, whether these altered circRNAs are responsible for resistance enhancers such as MAPK signals has still not been studied $[109,155]$.

\section{CircRNAs in cancer chemoresistance}

\section{Musculoskeletal system cancer: osteosarcoma}

Eighty circRNAs were identified as differentially expressed between chemoresistant and chemosensitive OS cells via circRNA profiling assays (20,836 circRNAs). Bioinformatics analysis showed that the altered circRNAs in chemoresistant OS cells were involved in several vital signaling pathways responsible for drug resistance, such as pathways featuring $\mathrm{ABC}$ transporters and VEGF. Hsa_circ_0001258 is decreased while hsa circ_0004674 is increased in chemoresistant OS cells and tissues [110, 112]. Hsa_circ_0001258 sponges miR744-3p to increase the expression of GSTM2. Inhibition of this axis induces resistance of OS cells to DOX [110]. Circ-LARP4 is downregulated in OS and negatively correlated with the Enneking stage and survival due to its ability to increase the sensitivity of tumor cells to cisplatin and DOX by sponging miR-424 [111].

Reduction of circ_001569 enhances the sensitivity of OS to cisplatin, DOX and methotrexate. Although circ_001569 overexpression increases the expression of $\mathrm{p}$-GSK-3 $\beta$ and $\beta$-catenin, the Wnt $/ \beta$-catenin agonist $\mathrm{LiCl}$ can restore the sensitivity of cancer cells to only cisplatin but not to DOX and methotrexate, suggesting that other mechanisms may be involved [138]. CircPVT1 is increased in OS tissues, serum, lung metastases and chemoresistant cells, which is correlated with poor prognosis. Knockdown of CircPVT1 partially reverses the resistance of OS cells to DOX and cisplatin by decreasing the expression of MDR protein 1 (MDR1 or ABCB1). It is speculated that circPVT1 may act as a sponge of miRNAs such as miR-125 to contribute to chemoresistance, which has not yet been validated $[18,40]$. 


\section{Respiratory system cancer}

Nasopharyngeal carcinoma CircCRIM1 was upregulated in highly metastatic NPC cells and tissues with distant metastasis. NPC patients with higher level of circCRIM1 has poorer therapeutic responses to docetaxel-based therapy, overall survival, disease-free survival and distant metastasis-free survival rates. CircCRIM1 is able to sponge miR-422a to increase mRNA and protein levels of FOXQ1. Through this, circCRIM1 promotes NPC cell metastasis and EMT. Silencing of it significantly enhances inhibitory efficacy of paclitaxel on metastasis in NPC cells [113].

Lung cancer In osimertinib-, DOX- or paclitaxelresistant lung cancer cells, thousands of altered circRNAs have been identified (Table 2) [20, 114, 115]. Similar to its role in radioresistant cervical cancer cells, hsa_circ_0004015 is also increased and confers resistance to gefitinib by regulating miR-1183/PDPK1 in NSCLC cells [109, 116, 117]. Circ_0076305 and circFGFR1 (hsa_circ_0084003) are elevated while circ 0002483 is decreased in NSCLC cells, which trends are more significant in the cisplatin- or paclitaxel-resistant NSCLC cells, respectively [116, 119]. Binding of circ 0076305 to miR-296-5p upregulates STAT3 and promotes cisplatin resistance [119]. Circ_0002483 reduces cell sensitivity to paclitaxel via the miR-182-5p/GRB2/ FOXO1 axis [116]. Upregulation of hsa_circ_0071799 affects miR-141, while downregulation of hsa_circ 0091931 affects miR-34c-5p, both of which may mediate osimertinib resistance [20].

CircCCDC66 and hsa-circRNA-002178 are highly expressed in lung adenocarcinoma (LUAD) cells $[60,118]$. CircCCDC66 is positively regulated by FAK (a marker of EMT) and HGF/c-Met, while it is negatively regulated by the acetylcholine receptor nAchR7 $\alpha$. However, reduction of circCCDC66 does not affect the resistance of H1975 cells to gefitinib or erlotinib but inhibits EMT and reduces cell sensitivity to cisplatin [118]. Hsa-circRNA-002178 enhances PD-L1 expression by sponging miR-34 to induce T cell exhaustion. Furthermore, it could also be transferred into CD8+ $\mathrm{T}$ cells to induce PD-1 expression via exosomes [60]. The elevation of circFGFR1 reduces PD-1positive lung cancer cell and $\mathrm{CD} 8+\mathrm{T}$ cell frequency by regulating the miR-381-3p/C-X-C motif chemokine receptor 4 (CXCR4) axis, which is responsible for reduced sensitivity to anti-PD-1 antibodies [120].

CircSETD3 is increased in the plasma of gefitinibresistant NSCLC patients and cells. It could directly bind to miR-520 to increase the expression of ABCG2, leading to a decreased intracellular accumulation of gefitinib [36]. The expression levels of ciRS-7 and circPVT1 are increased in pemetrexed- and cisplatin-resistant lung cancer cells. They reduce the efficiency of neoadjuvant chemotherapy (pemetrexed and cisplatin) by inhibiting the EGFR/PI3K pathway and the miR-145-5p/multidrug resistance protein 1 (MRP1 or $\mathrm{ABCC} 1$ ) axis, respectively $[41,121]$.

CircRNA_101505 confers HCC cells with resistance to cisplatin by regulating miR-103 [31]. MiR-103 is downregulated in lung cancer cells, and upregulation of miR-103 enhances the sensitivity of NSCLC cells to gefitinib [156]. However, whether circRNA_101505 is involved in METand miR-103-conferred gefitinib resistance in lung cancer still needs further evaluation. Circular RNA epithelial splicing regulatory protein-1 (cESRP1) is decreased in clinical small cell lung cancer (SCLC) tissues and DOX- and cisplatin/etoposide-resistant lung cancer cells. cESRP1 sponges miR-93-5p and activates Smad7/p21-TGF 3 -mediated EMT. Silencing cESRP1 caused chemoresistance and poor prognosis in SCLC patients [115].

\section{Hematopoietic system cancer: leukemia}

The introduced fusion of circRNA M9 reduces the sensitivity of K562 cells to arsenic trioxide (ATO) [157]. Circ_100053 and circ_0009910 are increased in imatinib-resistant leukemia cells $[122,126]$. Circ_ 0009910 sponges miR-34a-5p to elevate ULK1 and trigger autophagy, thus contributing to the chemoresistance of CML [126]. As a transcriptional accessory of BCRABL1, circBA9.3 is positively correlated with BCR-ABL1 in imatinib-resistant patients. Introducing exogenous circBA9.3 into TKI-sensitive K562 cells induces the resistance of CML not only to imatinib (1st generation TKI) but also to nilotinib and dasatinib (2nd generation TKIs). This probably occurs through increased expression of ABL1 and BCR-ABL1 at the posttranscriptional level [125].

CircPAN3 is increased in DOX-resistant acute myeloid leukemia (AML) cells and bone marrow cells from relapsed patients, while several target miRNAs are decreased. CircPAN3 has the potential to activate AMPK and inhibit mTOR, which subsequently induces autophagy. Meanwhile, circPAN3 binding to miR-153-5p and miR-183-5 increases the activity of X-linked inhibitor of apoptosis protein (XIAP). Without affecting basal apoptosis, knockdown of circPAN3 restores the sensitivity of AML cells to chemotherapeutics [123, 124]. CircMYBL2 could directly interact with the PTBP1 protein, which subsequently promotes the expression of FLT3 kinase at the translational level and phosphorylation of FLT3 kinase. These effects are why silencing circMYBL2 reduces proliferation, promotes differentiation and enhances cell sensitivity to quizartinib in FLT3-ITD AML cells [24].

\section{Endocrine system cancer}

Thyroid carcinoma CircEIF6 is highly expressed in papillary thyroid carcinoma (PTC) tissues and negatively 
regulates miR-144-3p expression. Exposure of TPC1 and BHT101 cells to cisplatin further increases circEIF6 and decreases miR-144-3p expression. CircEIF6 upregulation promotes cisplatin-induced autophagy; thus, it enhances the resistance of PTC cells to cisplatin via the miR-1443 p/TGF- $\alpha$ axis [79].

Pancreatic cancer $\mathrm{Xu}$ et al. identified that 26 circRNAs were upregulated and 55 were downregulated in gemcitabine-resistant pancreatic cancer cells. Further analysis showed that several markedly changed circRNAs were potentially involved in oncogenic pathways such as the MAPK pathway [19]. Another study showed that two circRNAs (Table 2) were significantly increased while miR-145 was decreased in plasma from gemcitabinenonresponsive patients and in gemcitabine-resistant PANC-1 (PANC-1/GEM) cells. Knockdown of them circRNAs effectively restores the sensitivity of PANC-1/ GEM cells to gemcitabine, probably via miR-145 [127].

Cervical cancer CircRNA-MTO1 (hsa-circRNA007874) and hsa_circ_0023404 are upregulated in cervical tumor cells, and both promote tumorigenesis and chemoresistance to cisplatin [128, 129, 158]. Sponging of miR-6893 by circMTO1 induces S100A1 expression and autophagy, leading to reduced chemosensitivity [128].

Breast cancer Circular RNA angiomotin-like 1 (circAMOTL1) could decrease paclitaxel-induced apoptosis by enhancing AKT signaling [159]. Hsa_circ_0001839 (circKDM4C) is decreased while hsa_circRNA_0003218 (circBMPR2) is increased in breast cancer tissues, especially in metastatic or chemoresistant tissues [136, 137]. Silencing circKDM4C promotes EMT while decreases apoptosis and the sensitivity of breast cancer cells to DOX by sponging miR-548p [137]. Circ_0006528 is increased in DOX-resistant breast cancer cells, while silencing of it enhances cell chemosensitivity by increasing miR-7-5p and Raf1 expression [130].

The expression level of ciRS-7 and REGY are higher while that of miR-7 is lower in breast cancer than in normal tissues, which trends are significant following chemotherapy. CiRS-7 is negatively correlated with the outcome of chemotherapy in breast cancer, suggesting that ciRS-7 may contribute to therapy resistance [131]. Silencing ciRS-7 increases miR-7 and decreases REGY expression. Indeed, the ciRS-7/miR-7 axis could mediate both cisplatin and 5-FU resistance in breast cancer cells $[3,131,132]$. CircRNA-MTO1 is decreased in monastrol-resistant breast cancer cells. Overexpression of circRNA-MTO1 inhibits viability and restores cell sensitivity to monastrol by reducing Eg 5 translation and sequestering TRAF4 from the Eg5 gene [134]. Hsa_circ 0025202 enhances the sensitivity of breast cancer cells to tamoxifen as a sponge of miR-182-5p to upregulate FOXO3a at both the mRNA and protein levels [135]. CircBMPR2 is reduced in metastatic breast cancer cells. Moreover, its downregulation results in the resistance of tumor cells to tamoxifen due to the enhanced EMT mediated by the miR-553/ubiquitin-specific protease 4 (USP4) axis [136].

\section{Digestive system cancer}

Oral carcinoma Hsa_circ_0005379 is downregulated in oral carcinoma tissues, while its elevation reduces cell proliferation, induces apoptosis and enhances the sensitivity of cancer cells to cetuximab. This is probably attributed to the function of hsa_circ_0005379 as an upstream inhibitor of EGFR- and EMT-related signals [138].

Hepatocellular carcinoma Circ_0003418 and circRNA_ 101505 are decreased in HCC tissues, while circSMARCA5 is reduced in intrahepatic cholangiocarcinoma (ICC) $[31,160,161]$. Silencing circ_0003418 promotes cisplatin resistance, probably though miR-7- and miR-383mediated effects on the Wnt/ $\beta$-catenin pathway, but this mechanism still needs further investigation [161]. CircRNA_101505 is especially lower in cisplatin-resistant HCC cells than in sensitive cells [31]. HCC or ICC patients with a lower level of circRNA_101505 or circSMARCA5 have a shorter overall survival period than patients with higher levels of these circRNAs [31, 160]. Overexpression of circRNA_101505 increases its ability to sponge miR-103 and subsequently elevates the expression of the tumor suppressor oxidored-nitro domaincontaining protein 1 (NOR1), which sensitizes HCC cells to cisplatin-induced apoptosis [31]. The upregulation of circ-SMARCA5 could enhance the sensitivity of ICC cells to cisplatin and gemcitabine [160].

Gastric cancer Similar to its levels in OS, circPVT1 is also increased in paclitaxel-resistant GC tissues and cells. It confers paclitaxel resistance in GC cells by sponging miR-124-3p to upregulate zinc-finger E-box binding homeobox 1 (ZEB1) levels [141]. Downregulation of ciRS-7 in GC cells increases miR-7-5p levels and subsequently reduces REGy expression, by which the toxicity of diosbulbin-B is enhanced [162].

Hsa_circ_0000199, circFN1 (hsa_circ_0058147) and hsa_circ_0081143 are predictive biomarkers of the sensitivity of GC patients to cisplatin, and they are upregulated in cisplatin-resistant GC cells [84, 139]. CircFN1 reduces GC cell apoptosis by sponging miR-182-5p in vitro and in vivo [140]. In addition to its status in cisplatin-resistant OC cells, PIK3R1, a regulatory subunit of PI3K, is also increased in cisplatin-resistant cells. 
Hsa_circ_0000199 sponges miR-198 to upregulate PIK3R1 at both the mRNA and protein levels. This further activates $\mathrm{PI} 3 \mathrm{~K} / \mathrm{AKT} / \gamma \mathrm{H} 2 \mathrm{AX}$ pathway-mediated DNA repair and confers cells with cisplatin resistance [84, 163]. Silencing of hsa_circ_0081143 makes cells more sensitive to cisplatin, probably by releasing miR646, which reduces CDK6 in GC tissues [139].

Colorectal cancer Hundreds of altered circRNAs have been identified in CRC cells resistant to 5 -FU and oxaliplatin or the exosomes derived from these resistant cells (Table 2). The circRNAs contained in exosomes could be transferred into cocultured cells in vitro, indicating their potential to be transported into adjacent or distant cells to confer chemoresistance in vivo, but this mechanism still requires further evaluation [29, 142]. Bioinformatics analysis further showed that the altered circRNAmediated signals were enriched in several cancer-related pathways. For instance, hsa_circRNA_103306/miR-370$3 p$ has the potential to regulate drug metabolism, while has_circ_32883/miR-130b probably affects drug resistance via the PI3K/AKT pathway [142]. Hsa_circ_32883 and ciRS-122 (hsa_circ_0005963) are upregulated in chemoresistant CRC cells [142, 143]. CiRS-122 can be delivered into chemosensitive cells via oxaliplatin-resistant cells-secreted exosomes. Then, it promotes glycolysis and cell resistance to oxaliplatin by sponging miR-122 to upregulate the M2 isoform of pyruvate kinase (PKM2) level [143]. Circ_001680 is increased in CRC tissues. In addition to enhancing proliferation and migration, it also triggers irinotecan resistance via sponging miR-340 to reduce BMI1 transcription, which supports the population of CSCs [46].

\section{Urinary system cancer}

Renal cancer Hsa_circ_0035483 is one of the most highly expressed circRNAs in renal clear cell carcinoma (RCC) tissues and cells, while its downstream target miR-335 displays the opposite trend. This inverse pattern is more obvious in the cells treated with gemcitabine. It was further demonstrated that the expression of hsa_circ_0035483 facilitates gemcitabine-induced autophagy and enhances the resistance of RCC to gemcitabine by regulating the hsa-miR-335/cyclin B1 axis [144].

Bladder cancer CircELP3 is increased and hsa_circ 0000285 is decreased in bladder cancer tissues or serum. Downregulating circELP3 reduces the sphere-forming ability of bladder cancer stem-like cells under hypoxic conditions. Moreover, hsa_circ_0000285 is exceptionally lower in cisplatin-resistant patients than in cisplatinsensitive patients $[96,145]$. Contrary to its high expression in breast cancer, ciRS-7 is expressed at low levels in bladder cancer [131, 164]. After induced overexpression of exogenous ciRS-7 in bladder cancer cells, proliferation, invasion and migration are markedly reduced, while cisplatin sensitivity is enhanced. This results from ciRS-7 acting as a sponge of miR-1270 to increase the mRNA level of apoptotic protease activating factor-1 (APAF1) [95].

Prostate cancer Recently, Greene et al. identified 278 circRNAs that were increased and 558 that were decreased in enzalutamide (ENZ)-resistant prostate cancer cells. Hsa_circ_0004870, derived from the RBM39 gene, is one of the significantly down-regulated circRNAs. It likely enhances chemoresistance by regulating the splicing of U2 small nuclear RNA auxiliary factor 65 (U2AF65) [147]. Silencing of circRNA Foxo3 (circFoxo3 or hsa_circis_0006404) increases the survival of androgen-enhanced prostate cancer cells and represses apoptosis while enhancing resistance to docetaxel. This is probably because the reduced activity of the circFoxo3/Foxo3 axis may lead to enhanced EMT [165]. CircRNA17 (hsa_circ_0001427), derived from the PDZ and LIM domain protein 5 (PDLIM5) gene, can increase and interact with miR-181c-5p to inhibit the transcription of androgen receptor variant 7 (ARv7). Due to ENZ-suppressed transcription of PDLIM5, circRNA17 is reduced in castration- and ENZ-resistant prostate cancer (CRPC) cells, which further grants cells resistance to ENZ [146].

\section{Reproductive system cancer: ovarian cancer}

Silencing of circ-ABCB10 promotes apoptosis of breast cancer cells and epithelial OC cells via decreased sponging of miR-1271, miR-1252 and miR-203 [38, 39]. Similar to its status in breast cancer, ciRS-7 is also decreased in tissues and serum exosomes derived from cisplatinresistant OC patients [26, 95]. Exogenous expression of ciRS-7 distinctly inhibits the proliferation and migration of cisplatin-resistant $\mathrm{OC}$ cells while promotes cisplatininduced apoptosis. CiRS-7 functions as a molecular sponge of miR-1270 to upregulate the transcription of suppressors of invasion (SCAI) [26]. Interestingly, circCELSR1 (hsa_circ_0063809), localized in the cytoplasm, is increased in paclitaxel-resistant OC tissues and cells, while miR-1252 is decreased in paclitaxel-resistant OC tissues and cells. It was further proven that circCELSR1 is negatively correlated with paclitaxel chemosensitivity by positively regulating FOXR2 expression by sponging miR-1252 [149].

\section{Miscellaneous: myeloma and melanoma}

Hsa_circ_0007841 is upregulated in bortezomib-resistant multiple myeloma (MM) cells and significantly correlated with the poor prognosis of MM patients [150]. CiRS-7 is downregulated in melanoma and negatively 
correlates with melanoma progression by interacting with the RBP IGF2BP3. Melanoma cells with lower levels of ciRS-7 are more sensitive to multiple MAPK pathway inhibitors and GPX4 inhibitors. However, silencing of ciRS-7 in melanoma cells with high ciRS-7 expression had no effect on cell sensitivity to the BRAF inhibitor dabrafenib and the GPX4 inhibitor RSL3 (1S,3R-RSL-3), suggesting that ciRS-7 levels are only a biomarker of chemotherapy response but not a critical mediator in melanoma [151].

\section{Conclusions and prospects}

Radiotherapy and chemotherapy are two widely used strategies in cancer treatment. However, intrinsic and acquired resistance are still critical obstacles for therapy outcome. CircRNAs are tissue- and cell type-specific, as well as cancer- and drug/radiation response-specific, leading to different expression patterns of circRNAs and associated regulatory pathways upon therapy stress. With high distinct abundance and stability, circRNAs are promising targets for overcoming cancer resistance to radiation and chemotherapy.

Currently, research on the role of circRNAs in the resistance of cancers to radiotherapy and chemotherapy is still at the nascent stage. First, the available reports about circRNAs in cancer chemoresistance are limited to only a few classic drugs and a few kinds of cancers. Second, most of the reported circRNAs, even when restricted to circRNAs identified in cancer, still have not been studied in cancer therapy responses, especially in radioresistance, and countless unidentified circRNAs have yet to be studied. These limitations will be overcome gradually, and potential interventions targeting circRNAs are promising for overcoming the resistance of cancer to radiation and chemotherapy.

\footnotetext{
Abbreviations

ABC: ATP-binding cassette; AML: Acute myeloid leukemia; APAF1: Apoptotic protease activating factor-1; AR: Androgen receptor; BBB: Blood-brain barrier; CESRP1: Circular RNA epithelial splicing regulatory protein 1; circRNAs: Circular RNAs; CRC: Colorectal cancer; CRPC: Castration-resistant prostate cancer; DOX: Doxorubicin; DYNLL1: Dynein light chain 1 protein; ecDNA: Extrachromosomal DNA; EGFR: Epidermal growth factor receptor; ENZ: Enzalutamide; ER: Endoplasmic reticulum; ESCC: Esophageal squamous cell carcinoma; EMT: Epithelial-to-mesenchymal transition; ecircRNAs: Exonic circRNAs; GC: Gastric cancer; H2AX: H2A histone family member X; HCC: Hepatocellular carcinoma; HR: Homologous recombination; ICC: Intrahepatic cholangiocarcinoma; LUAD: Lung adenocarcinoma; LncRNAs: Long ncRNAs; LRRC8: Leucine-rich repeat-containing protein 8C; MDR: Multidrug resistance; MDR1: MDR protein 1; miRNAs: MicroRNAs; MMR: Mismatch repair; MRP1: Multidrug resistance protein 1; ncRNAs: Noncoding RNAs; NK: Natural killer; NOR1: Oxidored-nitro domain-containing protein 1; NPC: Nasopharyngeal carcinoma; NSCLC: Non-small cell lung cancer; OC: Ovarian cancer; OS: Osteosarcoma; OSCC: Oral squamous cell carcinoma; PARP: Poly ADP-ribose polymerase; PDAC: Pancreatic ductal adenocarcinoma; PDLIM5: PDZ and LIM domain protein 5; PKC- : Protein kinase C $\varepsilon$; PKM2: M2 isoform of pyruvate kinase; PTBP1: Polypyrimidine tract binding protein 1; RBPs: RNA-binding proteins; RCC: Renal clear cell carcinoma; RTKs: Receptor tyrosine kinases; SCLC: Small cell lung cancer; SRC: Sarcoma viral oncogene homolog; TKIs: Tyrosine kinase inhibitors; TME: Tumor microenvironment;
}

TMZ: Temozolomide; U2AF65: U2 small nuclear RNA auxiliary factor 65; UPR: Unfolded protein response; USP4: Ubiquitin-specific protease 4; VRACs: Volume-regulated anion channels; XIAP: X-linked inhibitor of apoptosis protein; ZEB1: Zinc-finger E-box binding homeobox 1

\section{Acknowledgements}

Not applicable.

\section{Authors' contributions}

CCC and JBY collected the related reports and drafted the manuscript. $X \mathrm{~L}$, DLL, LWF and XWW revised the manuscript. LWF and XWW participated in designing the review. All authors read and approved the final manuscript.

\section{Funding}

This work is supported by the National Natural Science Foundation of China (No. 81902442) and the start-up funds of Xinxiang Medical University (No. 505284)

\section{Availability of data and materials}

Not applicable.

\section{Ethics approval and consent to participate}

Not applicable.

\section{Consent for publication}

Not applicable.

\section{Competing interests}

The authors declare that they have no competing interests.

\section{Author details}

${ }^{1}$ Henan Key Laboratory of Medical Tissue Regeneration, College of Basic Medical Sciences, Xinxiang Medical University, Xinxiang, Henan, China. ${ }^{2}$ School of Laboratory Medicine, Xinxiang Medical University, Xinxiang, Henan, China. ${ }^{3}$ State Key Laboratory of Oncology in South China;

Collaborative Innovation Center for Cancer Medicine, Sun Yat-sen University Cancer Center, Guangzhou, China.

Received: 9 December 2019 Accepted: 5 March 2020

Published online: 14 March 2020

\section{References}

1. Cocquerelle $C$, et al. Mis-splicing yields circular RNA molecules. FASEB J. 1993;7(1):155-60.

2. Sanger $\mathrm{HL}$, et al. Viroids are single-stranded covalently closed circular RNA molecules existing as highly base-paired rod-like structures. Proc Natl Acad Sci U S A. 1976;73(11):3852-6.

3. Memczak S, et al. Circular RNAs are a large class of animal RNAs with regulatory potency. Nature. 2013;495(7441):333-8.

4. Salzman J, et al. Circular RNAs are the predominant transcript isoform from hundreds of human genes in diverse cell types. PLoS One. 2012;7(2):e30733.

5. Haddad G, Lorenzen JM. Biogenesis and function of circular RNAs in health and in disease. Front Pharmacol. 2019;10:428.

6. Zhou R, et al. Circular RNAs (circRNAs) in cancer. Cancer Lett. 2018;425:13442.

7. Rastgoo N, et al. Role of epigenetics-microRNA axis in drug resistance of multiple myeloma. J Hematol Oncol. 2017;10(1):121.

8. Deng $\mathrm{H}$, et al. Role of long non-coding RNA in tumor drug resistance. Tumour Biol. 2016;37(9):11623-31.

9. Wang Y, et al. Long-noncoding RNAs (IncRNAs) in drug metabolism and disposition, implications in cancer chemo-resistance. Acta Pharm Sin B. 2020;10(1):105-12

10. An $X$, et al. Regulation of multidrug resistance by microRNAs in anti-cancer therapy. Acta Pharm Sin B. 2017;7(1):38-51.

11. Hanahan D, Weinberg RA. Hallmarks of cancer: the next generation. Cell. 2011;144(5):646-74.

12. Saxena M, et al. Transcription factors that mediate epithelial-mesenchymal transition lead to multidrug resistance by upregulating $A B C$ transporters. Cell Death Dis. 2011;2:e179.

13. Dean M, Fojo T, Bates S. Tumour stem cells and drug resistance. Nat Rev Cancer. 2005;5(4):275-84 
14. Tonnessen-Murray CA, et al. Chemotherapy-induced senescent cancer cells engulf other cells to enhance their survival. J Cell Biol. 2019;218(11):3827-44

15. Li C, et al. SKP2 promotes breast cancer tumorigenesis and radiation tolerance through PDCD4 ubiquitination. J Exp Clin Cancer Res. 2019;38(1):76.

16. Schulz A, et al. Cancer stem cells and radioresistance: DNA repair and beyond. Cancers (Basel). 2019:11(6):862.

17. Kristensen LS, et al. The biogenesis, biology and characterization of circular RNAs. Nat Rev Genet. 2019;20(11):675-91.

18. Chen J, et al. Circular RNA profile identifies circPVT1 as a proliferative factor and prognostic marker in gastric cancer. Cancer Lett. 2017;388:208-19.

19. Xu C, Yu Y, Ding F. Microarray analysis of circular RNA expression profiles associated with gemcitabine resistance in pancreatic cancer cells. Oncol Rep. 2018;40(1):395-404

20. Xu N, et al. Profiles and bioinformatics analysis of differentially expressed circRNAs in taxol-resistant non-small cell lung cancer cells. Cell Physiol Biochem. 2018;48(5):2046-60

21. Hansen TB, et al. Natural RNA circles function as efficient microRNA sponges. Nature. 2013;495(7441):384-8.

22. Tay Y, Rinn J, Pandolfi PP. The multilayered complexity of ceRNA crosstalk and competition. Nature. 2014;505(7483):344-52.

23. Wang $L$, et al. Circular RNA circRHOT1 promotes hepatocellular carcinoma progression by initiation of NR2F6 expression. Mol Cancer. 2019;18(1):119.

24. Sun YM, et al. circMYBL2, a circRNA from MYBL2, regulates FLT3 translation by recruiting PTBP1 to promote FLT3-ITD AML progression. Blood. 2019; 134(18):1533-46.

25. Xia X, et al. A novel tumor suppressor protein encoded by circular AKT3 RNA inhibits glioblastoma tumorigenicity by competing with active phosphoinositide-dependent Kinase-1. Mol Cancer. 2019;18(1):131

26. Zhao Z, et al. Circular RNA Cdrlas upregulates SCAl to suppress cisplatin resistance in ovarian cancer via miR-1270 suppression. Mol Ther Nucleic Acids. 2019;18(undefined):24-33.

27. Du WW, et al. Foxo3 circular RNA retards cell cycle progression via forming ternary complexes with p21 and CDK2. Nucleic Acids Res. 2016;44(6):2846-58

28. Ojha $\mathrm{R}$, et al. Emerging role of circular RNAs as potential biomarkers for the diagnosis of human diseases. Adv Exp Med Biol. 2018;1087(undefined):141-57.

29. Hon KW, et al. Extracellular vesicle-derived circular RNAs confers chemoresistance in colorectal cancer. Sci Rep. 2019;9(1):16497.

30. Huang $X-Y$, et al. Exosomal circRNA-100338 promotes hepatocellular carcinoma metastasis via enhancing invasiveness and angiogenesis. J Exp Clin Cancer Res. 2020;39(1):20.

31. Luo Y, et al. CircRNA_101505 sensitizes hepatocellular carcinoma cells to cisplatin by sponging miR-103 and promotes oxidored-nitro domaincontaining protein 1 expression. Cell Death Dis. 2019;5:121.

32. Joyce $\mathrm{H}$, et al. Influence of multidrug resistance and drug transport proteins on chemotherapy drug metabolism. Expert Opin Drug Metab Toxicol. 2015; 11(5):795-809.

33. Planells-Cases $R$, et al. Subunit composition of VRAC channels determines substrate specificity and cellular resistance to Pt-based anti-cancer drugs. EMBO J. 2015;34(24):2993-3008.

34. Gong J, et al. Microparticle drug sequestration provides a parallel pathway in the acquisition of cancer drug resistance. Eur J Pharmacol. 2013;721(1-3):116-25.

35. Wu S, Fu L. Tyrosine kinase inhibitors enhanced the efficacy of conventional chemotherapeutic agent in multidrug resistant cancer cells. Mol Cancer. 2018;17(1):25.

36. Dai $Y$, et al. CircSETD3 contributes to acquired resistance to gefitinib in nonsmall cell lung cancer by targeting miR-520h/ABCG2 pathway. Lancet. 2019; Available at SSRN: https://ssrn.com/abstract=3405549. Accessed 26 Sept 2019.

37. Wang XK, Fu LW. Interaction of tyrosine kinase inhibitors with the MDRrelated ABC transporter proteins. Curr Drug Metab. 2010;11(7):618-28.

38. Liang HF, et al. Circular RNA circ-ABCB10 promotes breast cancer proliferation and progression through sponging miR-1271. Am J Cancer Res. 2017;7(7):1566-76.

39. Chen $Y$, et al. Circular RNA ABCB10 correlates with advanced clinicopathological features and unfavorable survival, and promotes cell proliferation while reduces cell apoptosis in epithelial ovarian cancer. Cancer Biomark. 2019;26(2):151-61.

40. Kun-Peng Z, Xiao-Long M, Chun-Lin Z. Overexpressed circPVT1, a potential new circular RNA biomarker, contributes to doxorubicin and cisplatin resistance of osteosarcoma cells by regulating ABCB1. Int J Biol Sci. 2018; 14(3):321-30.
41. Zheng F, Xu R. CircPVT1 contributes to chemotherapy resistance of lung adenocarcinoma through miR-145-5p/ABCC1 axis. Biomed Pharmacother. 2020;124:109828.

42. Gately DP, Howell SB. Cellular accumulation of the anticancer agent cisplatin: a review. Br J Cancer. 1993;67(6):1171-6.

43. Xu B, et al. CircRNA CDR1as/miR-7 signals promote tumor growth of osteosarcoma with a potential therapeutic and diagnostic value. Cancer Manag Res. 2018;10:4871-80.

44. Sang M, et al. Circular RNA ciRS-7 accelerates ESCC progression through acting as a miR-876-5p sponge to enhance MAGE-A family expression. Cancer Lett. 2018;426:37-46.

45. Zhang $X$, et al. circSMAD2 inhibits the epithelial-mesenchymal transition by targeting miR-629 in hepatocellular carcinoma. Onco Targets Ther. 2018;11: 2853-63.

46. Jian $\mathrm{X}$, et al. Hsa circ 001680 affects the proliferation and migration of CRC and mediates its chemoresistance by regulating BMI1 through miR-340. Mol Cancer. 2020;19(1):20.

47. Zhao X, et al. miR-449c-5p availability is antagonized by circ-NOTCH1 for MYC-induced NOTCH1 upregulation as well as tumor metastasis and stemness in gastric cancer. J Cell Biochem. 2020. https://doi.org/10.1002/jcb. 29575.

48. Shibue T, Weinberg RA. EMT, CSCS, and drug resistance: the mechanistic link and clinical implications. Nat Rev Clin Oncol. 2017;14(10):611-29.

49. Mani SA, et al. The epithelial-mesenchymal transition generates cells with properties of stem cells. Cell. 2008;133(4):704-15.

50. Milanovic $M$, et al. Senescence-associated reprogramming promotes cancer stemness. Nature. 2018;553(7686):96-100.

51. Chen J, et al. circPTN sponges miR-145-5p/miR-330-5p to promote proliferation and stemness in glioma. J Exp Clin Cancer Res. 2019;38(1):398.

52. Han L, et al. Cancer stem cells: therapeutic implications and perspectives in cancer therapy. Acta Pharm Sin B. 2013;3(2):65-75.

53. Losic B, et al. Intratumoral heterogeneity and clonal evolution in liver cancer. Nat Commun. 2020;11(1):291.

54. Patel AP, et al. Single-cell RNA-seq highlights intratumoral heterogeneity in primary glioblastoma. Science. 2014;344(6190):1396-401.

55. Basile KJ, et al. In vivo MAPK reporting reveals the heterogeneity in tumoral selection of resistance to RAF inhibitors. Cancer Res. 2013;73(23):7101-10.

56. Lee HJ, et al. HER2 heterogeneity affects trastuzumab responses and survival in patients with HER2-positive metastatic breast cancer. Am J Clin Pathol. 2014;142(6):755-66.

57. Wolf $Y$, et al. UVB-induced tumor heterogeneity diminishes immune response in melanoma. Cell. 2019;179(1):219-235 e21.

58. $\mathrm{Ma} \mathrm{L}_{\text {, et }}$ al. Tumor cell biodiversity drives microenvironmental reprogramming in liver cancer. Cancer Cell. 2019;36(4):418-430 e6.

59. Luo F, et al. Niclosamide, an antihelmintic drug, enhances efficacy of PD-1/ PD-L1 immune checkpoint blockade in non-small cell lung cancer. J Immunother Cancer. 2019:7(1):245.

60. Wang J, et al. circRNA-002178 act as a ceRNA to promote PDL1/PD1 expression in lung adenocarcinoma. Cell Death Dis. 2020;11(1):32.

61. Tanaka $E$, et al. Expression of circular RNA CDR1AS in colon cancer cells increases cell surface PDL1 protein levels. Oncol Rep. 2019;42(4):1459-66.

62. Morales $C$, et al. Genetic determinants of methotrexate responsiveness and resistance in colon cancer cells. Oncogene. 2005:24(45):6842-7.

63. Nathanson DA, et al. Targeted therapy resistance mediated by dynamic regulation of extrachromosomal mutant EGFR DNA. Science. 2014;343(6166):72-6.

64. Huw LY, et al. Acquired PIK3CA amplification causes resistance to selective phosphoinositide 3-kinase inhibitors in breast cancer. Oncogenesis. 2013;2:e83.

65. Taniguchi $\mathrm{H}$, et al. AXL confers intrinsic resistance to osimertinib and advances the emergence of tolerant cells. Nat Commun. 2019;10(1):259.

66. Elkabets $\mathrm{M}$, et al. AXL mediates resistance to PI3Kalpha inhibition by activating the EGFR/PKC/mTOR axis in head and neck and esophageal squamous cell carcinomas. Cancer Cell. 2015:27(4):533-46.

67. Chen Y-f, Fu L-W. Mechanisms of acquired resistance to tyrosine kinase inhibitors. Acta Pharm Sin B. 2011;1(4):197-207.

68. Lee $\mathrm{HJ}$, et al. Drug resistance via feedback activation of Stat3 in oncogeneaddicted cancer cells. Cancer Cell. 2014;26(2):207-21.

69. Jacobsen $\mathrm{K}$, et al. Convergent Akt activation drives acquired EGFR inhibitor resistance in lung cancer. Nat Commun. 2017:8(1):410.

70. Wu J, et al. Tunicamycin specifically aggravates ER stress and overcomes chemoresistance in multidrug-resistant gastric cancer cells by inhibiting $\mathrm{N}$ glycosylation. J Exp Clin Cancer Res. 2018;37(1):272. 
71. Xu Z, et al. Inhibition of ER stress-related IRE1alpha/CREB/NLRP1 pathway promotes the apoptosis of human chronic myelogenous leukemia cell. Mol Immunol. 2018;101:377-85.

72. Mazumder A, et al. Hydroxycoumarin OT-55 kills CML cells alone or in synergy with imatinib or Synribo: involvement of ER stress and DAMP release. Cancer Lett. 2018;438:197-218.

73. $\mathrm{Ma} \mathrm{XH}$, et al. Targeting ER stress-induced autophagy overcomes BRAF inhibitor resistance in melanoma. J Clin Invest. 2014;124(3):1406-17.

74. Gupta A, et al. Autophagy inhibition and antimalarials promote cell death in gastrointestinal stromal tumor (GIST). Proc Natl Acad Sci U S A. 2010;107(32): 14333-8.

75. Smith AG, Macleod KF. Autophagy, cancer stem cells and drug resistance. J Pathol. 2019;247(5):708-18.

76. Bristol ML, et al. Dual functions of autophagy in the response of breast tumor cells to radiation: cytoprotective autophagy with radiation alone and cytotoxic autophagy in radiosensitization by vitamin D 3. Autophagy. 2012; 8(5):739-53.

77. Sheng Y, et al. DMH1 (4-[6-(4-isopropoxyphenyl)pyrazolo[1,5-a]pyrimidin-3yl]quinoline) inhibits chemotherapeutic drug-induced autophagy. Acta Pharm Sin B. 2015;5(4):330-6.

78. Tian $Y$, et al. ER-residential Nogo-B accelerates NAFLD-associated HCC mediated by metabolic reprogramming of oxLDL lipophagy. Nat Commun. 2019;10(1):3391.

79. Liu F, et al. Circular RNA EIF6 (Hsa_circ_0060060) sponges miR-144-3p to promote the cisplatin-resistance of human thyroid carcinoma cells by autophagy regulation. Aging (Albany NY). 2018;10(12):3806-20.

80. Burki TK. BRCA1 and BRCA2 mutation type associated with cancer risk. Lancet Oncol. 2015;16(5):e205.

81. Zhu $Y$, et al. Tamoxifen-resistant breast cancer cells are resistant to DNAdamaging chemotherapy because of upregulated BARD1 and BRCA1. Nat Commun. 2018;9(1):1595.

82. Kaplan AR, et al. Cediranib suppresses homology-directed DNA repair through down-regulation of BRCA1/2 and RAD51. Sci Transl Med. 2019; 11(492):eaav4508.

83. He YJ, et al. DYNLL1 binds to MRE11 to limit DNA end resection in BRCA1deficient cells. Nature. 2018;563(7732):522-6.

84. Huang $X$, et al. Circular RNA AKT3 upregulates PIK3R1 to enhance cisplatin resistance in gastric cancer via miR-198 suppression. Mol Cancer. 2019;18(1):71.

85. Russo M, et al. Adaptive mutability of colorectal cancers in response to targeted therapies. Science. 2019;366(6472):1473-80.

86. Jiao $\mathrm{S}$, et al. Differences in tumor microenvironment dictate $T$ helper lineage polarization and response to immune checkpoint therapy. Cell. 2019;179(5): 1177-1190 e13.

87. Ke $B$, et al. Interleukin-7 resensitizes non-small-cell lung cancer to cisplatin via inhibition of ABCG2. Mediat Inflamm. 2019;2019:7241418.

88. Zheng $X$, et al. Mitochondrial fragmentation limits NK cell-based tumor immunosurveillance. Nat Immunol. 2019;20(12):1656-67.

89. Tan Z, et al. PRSS contributes to cetuximab resistance in colorectal cancer. Sci Adv. 2020;6(1):eaax5576.

90. Choy ATF, et al. The microbiome of pancreatic cancer: from molecular diagnostics to new therapeutic approaches to overcome chemoresistance caused by metabolic inactivation of gemcitabine. Expert Rev Mol Diagn. 2018;18(12):1005-9.

91. Rafat M, et al. Macrophages promote circulating tumor cell-mediated local recurrence following radiotherapy in immunosuppressed patients. Cancer Res. 2018;78(15):4241-52.

92. Li X, et al. Reverse of microtubule-directed chemotherapeutic drugs resistance induced by cancer-associated fibroblasts in breast cancer. Onco Targets Ther. 2019;12:7963-73.

93. Ma Y, et al. Activated gastric cancer-associated fibroblasts contribute to the malignant phenotype and 5-FU resistance via paracrine action in gastric cancer. Cancer Cell Int. 2018;18(1):104.

94. Zou Y, et al. The role of circular RNA CDR1as/ciRS-7 in regulating tumor microenvironment: a pan-cancer analysis. Biomolecules. 2019; 9(9):429.

95. Yuan W, et al. Circular RNA Cdr1as sensitizes bladder cancer to cisplatin by upregulating APAF1 expression through miR-1270 inhibition. Mol Oncol. 2019;13(7):1559-76.

96. Su Y, et al. Hypoxia-elevated circELP3 contributes to bladder cancer progression and cisplatin resistance. Int J Biol Sci. 2019;15(2):441-52.
97. Wang $F$, et al. circRNA CDR1as regulated the proliferation of human periodontal ligament stem cells under a lipopolysaccharide-induced inflammatory condition. Mediat Inflamm. 2019;2019:1625381.

98. Zhang Q, et al. Roles of circRNAs in the tumour microenvironment. Mol Cancer. 2020;19(1):14.

99. Zhao M et al. Expression profiles and potential functions of circular RNAs in extracellular vesicles isolated from radioresistant glioma cells. Oncol Rep. 2019;41(3):1893-900.

100. Yang J, et al. Curcumin enhances radiosensitization of nasopharyngeal carcinoma by regulating circRNA network. Mol Carcinog. 2020;59(2):202..

101. Chen L, Zhou H, Guan Z. CircRNA_000543 knockdown sensitizes nasopharyngeal carcinoma to irradiation by targeting miR-9/platelet-derived growth factor receptor B axis. Biochem Biophys Res Commun. 2019;512(4): 786-92.

102. Chen $\mathrm{G}$, et al. Upregulation of circular RNA circATRNL1 to sensitize oral squamous cell carcinoma to irradiation. Mol Ther Nucleic Acids. 2020;19: 961-73.

103. Su H, et al. Profiling and bioinformatics analyses reveal differential circular RNA expression in radioresistant esophageal cancer cells. J Transl Med. 2016;14(1):225.

104. He $Y$, et al. CircVRK1 regulates tumor progression and radioresistance in esophageal squamous cell carcinoma by regulating miR-624-3p/PTEN/PI3K AKT signaling pathway. Int J Biol Macromol. 2019;125:116-23.

105. Liu J, et al. CircRNA_100367 regulated the radiation sensitivity of esophageal squamous cell carcinomas through miR-217/Wnt3 pathway. Aging (Albany NY). 2019;11:12412.

106. Yang W, et al. Knockdown of cZNF292 suppressed hypoxic human hepatoma SMMC7721 cell proliferation, vasculogenic mimicry, and radioresistance. Cell Signal. 2019;60:122-35.

107. Xiong W, et al. Microarray analysis of circular RNA expression profile associated with 5-fluorouracil-based chemoradiation resistance in colorectal cancer cells. Biomed Res Int. 2017;2017:8421614.

108. Wang L, et al. Inhibition of hsa_circ_0001313 (circCCDC66) induction enhances the radio-sensitivity of colon cancer cells via tumor suppressor miR-338-3p: effects of cicr_0001313 on colon cancer radio-sensitivity. Pathol Res Pract. 2019;215(4):689-96.

109. Yu D, et al. Comprehensive circular RNA expression profile in radiationtreated HeLa cells and analysis of radioresistance-related circRNAs. PeerJ. 2018;6:e5011.

110. Zhu KP, et al. Analyzing the interactions of mRNAs and ncRNAs to predict competing endogenous RNA networks in osteosarcoma chemo-resistance. Mol Ther. 2019;27(3):518-30

111. Hu Y, et al. Circular RNA LARP4 correlates with decreased Enneking stage, better histological response, and prolonged survival profiles, and it elevates chemosensitivity to cisplatin and doxorubicin via sponging microRNA-424 in osteosarcoma. J Clin Lab Anal. 2020;34(2):e23045.

112. Kun-Peng $Z$, et al. Screening circular RNA related to chemotherapeutic resistance in osteosarcoma by RNA sequencing. Epigenomics. 2018;10(10): 1327-46.

113. Hong $X$, et al. Circular RNA CRIM1 functions as a ceRNA to promote nasopharyngeal carcinoma metastasis and docetaxel chemoresistance through upregulating FOXQ1. Mol Cancer. 2020;19(1):33.

114. Chen T, et al. Comprehensive analysis of circular RNA profiling in AZD9291resistant non-small cell lung cancer cell lines. Thorac Cancer. 2019;10(4): 930-41.

115. Huang W, et al. Circular RNA cESRP1 sensitises small cell lung cancer cells to chemotherapy by sponging miR-93-5p to inhibit TGF-beta signalling. Cell Death Differ. 2019. https://doi.org/10.1038/s41418-019-0455-X.

116. Li X, et al. Hsa_circ_0002483 inhibited the progression and enhanced the Taxol sensitivity of non-small cell lung cancer by targeting miR-182-5p. Cell Death Dis. 2019;10(12):953.

117. Zhou Y, et al. Circular RNA hsa_circ_0004015 regulates the proliferation, invasion, and TKI drug resistance of non-small cell lung cancer by miR1183/PDPK1 signaling pathway. Biochem Biophys Res Commun. 2019; 508(2):527-35

118. Joseph NA, et al. The role of HGF-MET pathway and CCDC66 cirRNA expression in EGFR resistance and epithelial-to-mesenchymal transition of lung adenocarcinoma cells. J Hematol Oncol. 2018;11(1):74.

119. Dong Y, et al. Circ_0076305 regulates cisplatin resistance of non-small cell lung cancer via positively modulating STAT3 by sponging miR-296-5p. Life Sci. 2019;239:116984. 
120. Zhang PF, et al. Circular RNA circFGFR1 promotes progression and anti-PD-1 resistance by sponging miR-381-3p in non-small cell lung cancer cells. Mol Cancer. 2019;18(1):179.

121. Mao Y, Xu R. Circular RNA CDR1-AS contributes to pemetrexed and cisplatin chemoresistance through EGFR/PI3K signaling pathway in lung adenocarcinoma. Biomed Pharmacother. 2019;123:109771.

122. Ping L, et al. High circ_100053 predicts a poor outcome for chronic myeloid leukemia and is involved in imatinib resistance. Oncol Res. 2019. https://doi. org/10.3727/096504018X15412701483326.

123. Shang J, et al. CircPAN3 contributes to drug resistance in acute myeloid leukemia through regulation of autophagy. Leuk Res. 2019;85:106198.

124. Shang J, et al. CircPAN3 mediates drug resistance in acute myeloid leukemia through the miR-153-5p/miR-183-5p-XIAP axis. Exp Hematol. 2019; 70:42-54 e3

125. Pan Y, et al. CircBA9.3 supports the survival of leukaemic cells by upregulating c-ABL1 or BCR-ABL1 protein levels. Blood Cells Mol Dis. 2018;73: 38-44.

126. Cao HX, et al. Circ_0009910 promotes imatinib resistance through ULK1induced autophagy by sponging miR-34a-5p in chronic myeloid leukemia. Life Sci. 2020;243:117255

127. Shao F, et al. Circular RNA signature predicts gemcitabine resistance of pancreatic ductal adenocarcinoma. Front Pharmacol. 2018;9:584.

128. Chen $\mathrm{M}$, et al. circMTO1 promotes tumorigenesis and chemoresistance of cervical cancer via regulating miR-6893. Biomed Pharmacother. 2019;117: 109064.

129. Guo J, et al. Hsa_circ_0023404 enhances cervical cancer metastasis and chemoresistance through VEGFA and autophagy signaling by sponging miR-5047. Biomed Pharmacother. 2019;115:108957.

130. Gao D, et al. Screening circular RNA related to chemotherapeutic resistance in breast cancer. Epigenomics. 2017;9(9):1175-88.

131. Yang $W$, et al. Silencing CDR1as enhances the sensitivity of breast cancer cells to drug resistance by acting as a miR-7 sponge to down-regulate REGgamma. J Cell Mol Med. 2019;23(8):4921-32.

132. Xu H, et al. The circular RNA Cdr1as, via miR-7 and its targets, regulates insulin transcription and secretion in islet cells. Sci Rep. 2015;5:12453.

133. Yang $L$, et al. The role of CDR1as in proliferation and differentiation of human umbilical cord-derived mesenchymal stem cells. Stem Cells Int. 2019;2019:2316834.

134. Liu Y, et al. Circular RNAMTO1 suppresses breast cancer cell viability and reverses monastrol resistance through regulating the TRAF4/Eg5 axis. Int J Oncol. 2018;53(4):1752-62.

135. Sang Y, et al. circRNA_0025202 regulates tamoxifen sensitivity and tumor progression via regulating the miR-182-5p/FOXO3a Axis in breast cancer. Mol Ther. 2019;27(9):1638-52.

136. Liang $Y$, et al. Targeting the circBMPR2/miR-553/USP4 axis as a potent therapeutic approach for breast cancer. Mol Ther Nucleic Acids. 2019;17: 347-61.

137. Liang $Y$, et al. circKDM4C suppresses tumor progression and attenuates doxorubicin resistance by regulating miR-548p/PBLD axis in breast cancer. Oncogene. 2019;38(42):6850-66.

138. Zhang $\mathrm{H}$, et al. Expression of circ_001569 is upregulated in osteosarcoma and promotes cell proliferation and cisplatin resistance by activating the Wnt/beta-catenin signaling pathway. Oncol Lett. 2018;16(5):5856-62.

139. Xue $M$, et al. hsa_circ_0081143 promotes cisplatin resistance in gastric cancer by targeting miR-646/CDK6 pathway. Cancer Cell Int. 2019;19:25.

140. Huang XX, et al. A novel circular RNA circFN1 enhances cisplatin resistance in gastric cancer via sponging miR-182-5p. J Cell Biochem. 2020.

141. Liu YY, Zhang LY, Du WZ. Circular RNA circ-PVT1 contributes to paclitaxel resistance of gastric cancer cells through the regulation of ZEB1 expression by sponging miR-124-3p. Biosci Rep. 2019;39(12):BSR20193045.

142. Abu N, et al. Identification of differentially expressed circular RNAs in chemoresistant colorectal cancer. Epigenomics. 2019;11(8):875-84

143. Wang $X$, et al. Exosome-delivered circRNA promotes glycolysis to induce chemoresistance through the miR-122-PKM2 axis in colorectal cancer. Mol Oncol. 2020. https://doi.org/10.1002/1878-0261.12629.

144. Yan L, et al. Hsa_circ_0035483 sponges hsa-miR-335 to promote the gemcitabine-resistance of human renal cancer cells by autophagy regulation. Biochem Biophys Res Commun. 2019;519(1):172-8.

145. Chi BJ, et al. Downregulation of hsa_circ_0000285 serves as a prognostic biomarker for bladder cancer and is involved in cisplatin resistance. Neoplasma. 2019;66(2):197-202.
146. Wu G, et al. Preclinical study using circular RNA 17 and micro RNA 181c-5p to suppress the enzalutamide-resistant prostate cancer progression. Cell Death Dis. 2019;10(2):37.

147. Greene J, et al. Circular RNAs are differentially expressed in prostate cancer and are potentially associated with resistance to enzalutamide. Sci Rep. 2019;9(1):10739.

148. Antonarakis ES, et al. AR-V7 and resistance to enzalutamide and abiraterone in prostate cancer. N Engl J Med. 2014;371(11):1028-38.

149. Zhang S, et al. circCELSR1 (hsa_circ_0063809) contributes to paclitaxel resistance of ovarian cancer cells by regulating FOXR2 expression via miR1252. Mol Ther. 2020;19:718-30.

150. Gao M, et al. hsa_circ_0007841: a novel potential biomarker and drug resistance for multiple myeloma. Front Oncol. 2019;9:1261.

151. Hanniford D, et al. Epigenetic silencing of CDR1as drives IGF2BP3-mediated melanoma invasion and metastasis. Cancer Cell. 2020;37(1):55-70.e15.

152. Wu P, et al. KHDRBS3 regulates the permeability of blood-tumor barrier via cDENND4C/miR-577 axis. Cell Death Dis. 2019;10(7):536.

153. Emons $\mathrm{G}$, et al. Chemoradiotherapy resistance in colorectal cancer cells is mediated by Wnt/beta-catenin signaling. Mol Cancer Res. 2017:15(11):1481-90.

154. He N, et al. Analysis of circular RNA expression profile in HEK 293T cells exposed to ionizing radiation. Dose-Response. 2019;17(2): 1559325819837795

155. Che Y, et al. TRIP4 promotes tumor growth and metastasis and regulates radiosensitivity of cervical cancer by activating MAPK, PI3K/AKT, and hTERT signaling. Cancer Lett. 2019;452:1-13.

156. Garofalo $M$, et al. EGFR and MET receptor tyrosine kinase-altered microRNA expression induces tumorigenesis and gefitinib resistance in lung cancers. Nat Med. 2011;18(1):74-82.

157. Guarnerio J, et al. Oncogenic role of fusion-circRNAs derived from cancerassociated chromosomal translocations. Cell. 2016;165(2):289-302.

158. Zhang J, et al. Circular RNA hsa_circ_0023404 exerts an oncogenic role in cervical cancer through regulating miR-136/TFCP2/YAP pathway. Biochem Biophys Res Commun. 2018;501(2):428-33.

159. Ma J, et al. Posttranscriptional regulation of AKT by circular RNA angiomotin- like 1 mediates chemoresistance against paclitaxel in breast cancer cells. Aging (Albany NY). 2019;11(23):11369-81.

160. Lu Q, Fang T. Circular RNA SMARCA5 correlates with favorable clinical tumor features and prognosis, and increases chemotherapy sensitivity in intrahepatic cholangiocarcinoma. J Clin Lab Anal. 2019:e23138. https:// onlinelibrary.wiley.com/doi/full/10.1002/jcla.23138.

161. Chen $\mathrm{H}$, et al. circ_0003418 inhibits tumorigenesis and cisplatin chemoresistance through Wnt/beta-catenin pathway in hepatocellular carcinoma. Onco Targets Ther. 2019;12:9539-49.

162. Li C, Li M, Xue Y. Downregulation of CircRNA CDR1as specifically triggered low-dose diosbulbin-B induced gastric cancer cell death by regulating miR7-5p/REGgamma axis. Biomed Pharmacother. 2019;120:109462.

163. Cheung LW, Mills GB. Targeting therapeutic liabilities engendered by PIK3R1 mutations for cancer treatment. Pharmacogenomics. 2016;17(3):297-307.

164. Li $P$, et al. CircRNA-Cdr1as exerts anti-oncogenic functions in bladder cancer by sponging microRNA-135a. Cell Physiol Biochem. 2018:46(4):1606-16.

165. Shen Z, et al. Reduction of circular RNA Foxo3 promotes prostate cancer progression and chemoresistance to docetaxel. Cancer Lett. 2020;468:88-101.

\section{Publisher's Note}

Springer Nature remains neutral with regard to jurisdictional claims in published maps and institutional affiliations.

Ready to submit your research? Choose BMC and benefit from:

- fast, convenient online submission

- thorough peer review by experienced researchers in your field

- rapid publication on acceptance

- support for research data, including large and complex data types

- gold Open Access which fosters wider collaboration and increased citations

- maximum visibility for your research: over $100 \mathrm{M}$ website views per year

At $\mathrm{BMC}$, research is always in progress.

Learn more biomedcentral.com/submission 